\title{
Stability and Stabilization of Delayed T-S Fuzzy Systems: A Delay Partitioning Approach
}

\author{
Yan Zhao, Huijun Gao, Member, IEEE, James Lam, Senior Member, IEEE, and Baozhu Du
}

\begin{abstract}
This paper proposes a new approach, namely, the delay partitioning approach, to solving the problems of stability analysis and stabilization for continuous time-delay TakagiSugeno fuzzy systems. Based on the idea of delay fractioning, a new method is proposed for the delay-dependent stability analysis of fuzzy time-delay systems. Due to the instrumental idea of delay partitioning, the proposed stability condition is much less conservative than most of the existing results. The conservatism reduction becomes more obvious with the partitioning getting thinner. Based on this, the problem of stabilization via the so-called parallel distributed compensation scheme is also solved. Both the stability and stabilization results are further extended to time-delay fuzzy systems with time-varying parameter uncertainties. All the results are formulated in the form of linear matrix inequalities (LMIs), which can be readily solved via standard numerical software. The advantage of the results proposed in this paper lies in their reduced conservatism, as shown via detailed illustrative examples. The idea of delay partitioning is well demonstrated to be efficient for conservatism reduction and could be extended to solving other problems related to fuzzy delay systems.
\end{abstract}

Index Terms-Delay partitioning, stability, Takagi-Sugeno (TS) fuzzy systems, time-delay systems, uncertainties.

\section{INTRODUCTION}

$\mathbf{S}$ INCE most physical systems and processes in the real world are nonlinear, researchers have been devoting their efforts to seeking an effective means of controlling nonlinear systems. Among the many developments, there are growing interests in the fuzzy control of complex nonlinear systems and, in particular, Takagi-Sugeno (T-S) fuzzy-model-based control [11], [21]. It has been proved that T-S fuzzy models can approximate any smooth nonlinear system to any accuracy on a compact set, which is realized by piecewise smoothly connecting a family of local linear models with fuzzy membership functions. This "blending" makes T-S fuzzy models similar to linear systems, and the stability analysis and synthesis can be derived by making full use of the fruitful results on linear systems. So far, a great number of results have been reported for T-S fuzzy systems. To

Manuscript received May 6, 2007; accepted June 28, 2007. First published July 16, 2008; current version published July 29, 2009. This work was supported in part by the National Natural Science Foundation of China (60825303, 60834003), in part by the 973 Project (2009CB320600), in part by the Research Fund for the Doctoral Programme of Higher Education of China (20070213084), in part by the Heilongjiang Outstanding Youth Science Fund (JC200809), in part by Postdoctoral Science Foundation of China (200801282), in part by the Fok Ying Tung Education Foundation (111064), and in part by the Research Grants Council under HKU 7031/07P.

H. Gao and Y. Zhao are with the Space Control and Inertial Technology Research Center, Harbin Institute of Technology, Harbin 150001, China (e-mail: hjgao@hit.edu.cn).

J. Lam and B. Du are with the Department of Mechanical Engineering, University of Hong Kong, Pok Fu Lam, Hong Kong (e-mail: james.lam@hku.hk).

Digital Object Identifier 10.1109/TFUZZ.2008.928598 mention a few, the problem of stability analysis is investigated in [19], [29], and [31]; stabilizing and $H_{\infty}$ control designs are reported in [4], [5], [15], [22], [28], [40], [45], and [47]; state estimation is addressed in [1], [10], and [46]; reliable control strategies are presented in [34] and [35]; and fault detection is considered in [25].

On the other hand, after-effect phenomena often appear in various engineering, communication, and chemical processes. In modern industry, the traditional point-to-point communication architecture is no longer efficient, as the physical setups and functionalities are continuously expanding, which pushes the introduction of communication network media due to advantages such as modularity, decentralization of control, integrated diagnostics, quick and easy maintenance, and low cost. As the hardware devices for networks and network nodes become cheaper, the insertion of networks into control loops becomes increasingly common. However, due to the time-sharing nature of the communication media, time delays inevitably appear in the control loops. In control systems, time delays often degrade the system's performance and even cause instability. Therefore, time delays have received great attention in recent years, and many researchers have studied various analytical techniques and developed many synthesis methods for time-delay systems. For instance, stability analysis is carried out in [18], [24], [39], and [43]; stabilizing and $H_{\infty}$ controllers are designed in [27] and [38]; model reduction is addressed in [41]; and filtering problems are investigated in [2], [13], [17], [33], and [44].

When nonlinearity is considered, T-S fuzzy systems with time delays have received great attention in recent years. Researchers have proposed various approaches for the analysis and synthesis of fuzzy time-delay systems [23], [32]. Generally speaking, these approaches are based on two theories. One is the Lyapunov-Krasovskii stability theory [14], [36], [47], which is widely used but needs much information about the time delay. The other is the Lyapunov-Razumikhin method [6], which does not need too much information about the time delay, but the obtained results may be conservative. Existing results can be roughly classified into two types: One is dependent on the size of the delay, i.e., the so-called "delay-dependent" approach [3], [9], [12], while the other is not concerned with the size of the delay, which is called as the "delay-independent" approach. It has been recognized that the former is less conservative than the latter. As the conservatism has been well recognized as one of the most important deficiencies in existing results, in recent years, a lot of attention has been devoted to reducing the overdesign via various methods. Among these efforts, the introduction of new types of Lyapunov functional [7], [36] and adoption of appropriate bounding techniques [7] have 
been the most common approaches that have been reported. It is worth mentioning, however, that the improvement due to the earlier methods is still limited, and how to further reduce the conservatism and overdesign still remains an important and challenging problem.

Motivated by these earlier developments, in this paper, we propose a new approach for the stability analysis and stabilization of T-S fuzzy time-delay systems. Our objective is to further reduce the conservatism and overdesign in the existing results, and the instrumental idea to achieve our goal is "delay partitioning." Based on this idea, a new method is first proposed for the delay-dependent stability analysis of fuzzy time-delay systems. The proposed stability condition is much less conservative than most of the existing results due to the delay partitioning, and it becomes even less conservative when the partitioning goes finer. Using this result, the problem of stabilization via the socalled parallel distributed compensation scheme is also solved. Both the stability and stabilization results are further extended to time-delay fuzzy systems with parameter uncertainties. All the results are formulated in the form of linear matrix inequalities (LMIs), which can be readily solved via standard numerical software. The merit of the results proposed in this paper lies in their reduced conservatism, as will be shown via detailed examples.

The remaining portion of the paper is organized as follows. Section II formulates the problem under consideration. Stability and robust stability analysis results are presented in Section III. Based on the results obtained in Section III, controller designs are presented in Section IV. Illustrative examples are given in Section V to demonstrate the effectiveness of the theoretical results. Finally, some concluding remarks are given in Section VI.

The notation used throughout the paper is fairly standard. The superscript " $T$ " stands for matrix transposition; $\mathbb{R}^{n}$ denotes the $n$-dimensional Euclidean space. The notation $\|\cdot\|$ refers to the Euclidean vector norm, and $\operatorname{diag}\{\ldots\}$ stands for a blockdiagonal matrix. In symmetric block matrices or complex matrix expressions, we use an asterisk $(*)$ to represent a term that is induced by symmetry. $I$ and 0 denote the identity matrix and zero matrix with compatible dimensions, respectively, and $\operatorname{sym}(A)$ is defined as $A+A^{T}$. $A-B>0$ means that $A-B$ is a real symmetric positive definite matrix. Matrices, if their dimensions are not explicitly stated, are assumed to be compatible for algebraic operations.

\section{PREliminaries}

Consider a nonlinear time-delay system that can be represented by the following T-S fuzzy time-delay model:

Plant rule: IF $\theta_{1}(t)$ is $M_{i 1}$ and $\theta_{2}(t)$ is $M_{i 2}$ and $\cdots$ and $\theta_{p}(t)$ is $M_{i p}$, THEN

$$
\left\{\begin{aligned}
\dot{x}(t)= & \left(A_{i}+\Delta A_{i}(t)\right) x(t)+\left(A_{d i}+\Delta A_{d i}(t)\right) x(t-h) \\
& +\left(B_{i}+\Delta B_{i}(t)\right) u(t), \quad t>0 \\
x(t)= & \varphi(t), \quad t \in[-\bar{h}, 0], \quad i=1,2, \ldots, r
\end{aligned}\right.
$$

where $x(t) \in \mathbb{R}^{n}$ is the state vector, $u(t) \in \mathbb{R}^{p}$ is the control input vector, $h$ is a constant time delay satisfying $0 \leq h \leq \bar{h}$, $\varphi(t)$ is the initial condition, $M_{i j}$ is the fuzzy set, $r$ is the number of IF-THEN rules, and $\theta(t)=\left[\theta_{1}(t), \quad \theta_{2}(t), \ldots, \theta_{p}(t)\right]$ is the premise variables vector. It is assumed that the premise variables do not depend on the input $u(t)$, and $A_{i}, B_{i}$, and $A_{d i}$ are known constant matrices with appropriate dimensions. $\Delta A_{i}(t)$, $\Delta A_{d i}(t)$, and $\Delta B_{i}(t)$ denote the uncertainties in the system and they are of the form [26]

$$
\begin{aligned}
& \Delta A_{i}(t)=D_{a i} F(t) E_{a i}, \quad \Delta A_{d i}(t)=D_{d i} F(t) E_{d i} \\
& \Delta B_{i}(t)=D_{b i} F(t) E_{b i}, \quad i=1,2, \ldots, r
\end{aligned}
$$

where $D_{a i}, D_{d i}, D_{b i}, E_{a i}, E_{d i}$, and $E_{b i}$ are known constant matrices and $F(t)$ is an unknown real time-varying matrix with Lebesgue measurable elements bounded by

$$
F^{T}(t) F(t) \leq I \text {. }
$$

Given a pair of $(x(t), u(t))$, the overall fuzzy system is inferred as

$$
\dot{x}(t)=\sum_{i=1}^{r} \lambda_{i}(\theta(t))\left[\tilde{A}_{i} x(t)+\tilde{A}_{d i} x(t-h)+\tilde{B}_{i} u(t)\right]
$$

where

$$
\begin{array}{r}
\tilde{A}_{i}=A_{i}+\Delta A_{i}(t), \quad \tilde{A}_{d i}=A_{d i}+\Delta A_{d i}(t) \\
\tilde{B}_{i}=B_{i}+\Delta B_{i}(t)
\end{array}
$$

and

$$
\begin{aligned}
\sum_{i=1}^{r} \lambda_{i}(\theta(t)) & =1 \\
\lambda_{i}(\theta(t)) & =\frac{\omega_{i}(\theta(t))}{\sum_{i=1}^{r} \omega_{i}(\theta(t))} \geq 0 \\
\omega_{i}(\theta(t)) & =\prod_{j=1}^{p} M_{i j}\left(\theta_{j}(t)\right)
\end{aligned}
$$

with $M_{i j}\left(\theta_{j}(t)\right)$ representing the grade of membership of $\theta_{j}(t)$ in $M_{i j}$. Then, it can be seen that

$$
\begin{aligned}
\omega_{i}(\theta(t)) & \geq 0, \quad i=1,2, \ldots, r \\
\sum_{i=1}^{r} \omega_{i}(\theta(t)) & >0
\end{aligned}
$$

for all $t$. In this paper, we will first consider the nominal fuzzy system of (1), which is given by

$$
\dot{x}(t)=\sum_{i=1}^{r} \lambda_{i}(\theta(t))\left(A_{i} x(t)+A_{d i} x(t-h)+B_{i} u(t)\right) .
$$

A more compact presentation of the T-S fuzzy model is given by

$$
\begin{aligned}
& \dot{x}(t)=A(t) x(t)+A_{d}(t) x(t-h)+B(t) u(t) \\
& x(t)=\varphi(t), \quad t \in[-\bar{h}, 0]
\end{aligned}
$$

where

$$
A(t)=\sum_{i=1}^{r} \lambda_{i}(\theta(t)) A_{i}, \quad A_{d}(t)=\sum_{i=1}^{r} \lambda_{i}(\theta(t)) A_{d i}
$$




$$
B(t)=\sum_{i=1}^{r} \lambda_{i}(\theta(t)) B_{i}
$$

The parallel distributed compensation strategy is utilized and the fuzzy state-feedback controller obeys the following rules.

Controller rule: IF $\theta_{1}(t)$ is $M_{i 1}$ and $\theta_{2}(t)$ is $M_{i 2}$ and $\cdots$ and $\theta_{p}(t)$ is $M_{i p}$, THEN

$$
\begin{aligned}
u(t) & =K_{i} x(t) \\
i & =1, \ldots, r
\end{aligned}
$$

where $x(t) \in \mathbb{R}^{n}$ is the input of the controller, $u(t) \in \mathbb{R}^{m}$ is the output of the controller, and $K_{i}$ is the gain matrix of the statefeedback controller. Thus, the controller can be represented by the following input-output form:

$$
u(t)=\sum_{i=1}^{r} \lambda_{i}(\theta(t)) K_{i} x(t)
$$

with its compact form

$$
u(t)=K(t) x(t)
$$

where

$$
K(t)=\sum_{i=1}^{r} \lambda_{i}(\theta(t)) K_{i} .
$$

Thus, the closed-loop system can be obtained as

$$
\begin{aligned}
\dot{x}(t)=\sum_{i=1}^{r} \sum_{j=1}^{r} \lambda_{i}(\theta(t)) \lambda_{j}(\theta(t))\left[\tilde{A}_{i} x(t)\right. & +\tilde{A}_{d i} x(t-h) \\
& \left.+\tilde{B}_{i} K_{j} x(t)\right] .
\end{aligned}
$$

The closed-loop system for the nominal case is given by

$$
\begin{array}{r}
\dot{x}(t)=\sum_{i=1}^{r} \sum_{j=1}^{r} \lambda_{i}(\theta(t)) \lambda_{j}(\theta(t))\left[A_{i} x(t)+\right. \\
A_{d i} x(t-h) \\
\left.+B_{i} K_{j} x(t)\right]
\end{array}
$$

and its compact form is given by

$$
\dot{x}(t)=[A(t)+B(t) K(t)] x(t)+A_{d}(t) x(t-h) .
$$

\section{Stability ANALYSIS}

In this section, by employing the instrumental idea of delay fractioning, where similar ideas have also appeared in [16] and [20], a new Lyapunov-Krasovskii functional candidate for T-S fuzzy time-delay systems is introduced. Based on this Lyapunov-Krasovskii functional candidate, a new delaydependent stability criterion is first derived for nominal fuzzy time-delay systems. Then, this result is further extended to obtain a new robust stability condition for fuzzy time-delay systems with parameter uncertainties.

We first introduce the following matrix functions, which will be used in the subsequent results:

$$
\begin{aligned}
& R(t)=\sum_{i=1}^{r} \lambda_{i}(\theta(t)) R_{i}>0, \quad Q(t)=\sum_{i=1}^{r} \lambda_{i}(\theta(t)) Q_{i}>0 \\
& Z(t)=\sum_{i=1}^{r} \lambda_{i}(\theta(t)) Z_{i}>0
\end{aligned}
$$

$$
S_{1}(t)=\sum_{i=1}^{r} \lambda_{i}(\theta(t)) S_{1 i}, \quad S_{2}(t)=\sum_{i=1}^{r} \lambda_{i}(\theta(t)) S_{2 i}
$$

where $R_{i}>0, Q_{i}>0, Z_{i}>0$, and $S_{1 i}$ and $S_{2 i}$ are constant matrices.

\section{A. Nominal Systems}

In this section, by assuming that the controller gain matrices in the controller (9) are given, a delay-dependent stability criterion for the nominal closed-loop system in (12) is presented in the following theorem.

Theorem 1: Consider the nominal fuzzy time-delay system in (7) and suppose that the controller gain matrices in (10) are known. Given an integer $m \geq 1$ and a scalar $h>0$, if there exist a matrix $P>0$ and matrix functions $Q(t)>0, Z(t)>0$, $R(t)>0, S_{1}(t)$, and $S_{2}(t)$ in the form of (14), such that the following inequalities are satisfied for any, $w$, and some positive scalar $\sigma$ :

$$
\left[\begin{array}{cc}
\Omega(t)+\sigma W_{\sigma}^{T} W_{\sigma} & S_{1}(t) \\
* & -\frac{m}{h} Z(t)
\end{array}\right]<0
$$

where

$$
\begin{aligned}
& \Omega(t)=W_{P}^{T} \bar{P} W_{P}+\frac{h}{m} W_{R}^{T} R(t) W_{R}+W_{Q_{1}}^{T} Q(t) W_{Q_{1}} \\
& -W_{Q_{2}}^{T} Q\left(t-\frac{h}{m}\right) W_{Q_{2}}+\operatorname{sym}\left(S(t) W_{S}(t)\right) \\
& \bar{P}=\left[\begin{array}{ll}
0 & P \\
P & 0
\end{array}\right], \quad S(t)=\left[\begin{array}{ll}
S_{1}(t) & S_{2}(t)
\end{array}\right] \\
& W_{P}=\left[\frac{I_{n} 0_{n,(m+1) n}}{0_{n,(m+1) n} I_{n}}\right], \quad W_{R}=\left[\begin{array}{ll}
0_{n,(m+1) n} & I_{n}
\end{array}\right] \\
& W_{Q_{1}}=\left[\begin{array}{ll}
I_{m n} & 0_{m n, 2 n}
\end{array}\right], \quad W_{\sigma}=\left[\begin{array}{ll}
I_{n} & 0_{n,(m+1) n}
\end{array}\right] \\
& W_{Q_{2}}=\left[\begin{array}{lll}
0_{m n, n} & I_{m n} & 0_{m n, n}
\end{array}\right] \\
& W_{S}(t)=\left[\frac{I_{n}-I_{n} 0_{n, m n}}{A(t)+B(t) K(t) 0_{n,(m-1) n} A_{d}(t)-I_{n}}\right]
\end{aligned}
$$

then the fuzzy system in (13) is asymptotically stable.

Proof: To prove the theorem, we choose a LyapunovKrasovskii functional candidate as

$$
V(t)=V_{1}(t)+V_{2}(t)+V_{3}(t)
$$

and

$$
\begin{aligned}
& V_{1}(t)=x^{T}(t) P x(t) \\
& V_{2}(t)=\int_{-h / m}^{0} \int_{t+v}^{t} \dot{x}^{T}(w) R(w) \dot{x}(w) d w d v \\
& V_{3}(t)=\int_{t-h / m}^{t} \gamma^{T}(w) Q(w) \gamma(w) d w
\end{aligned}
$$


where $P>0, R(t)>0$, and $Q(t)>0$ have been defined in (14), and

$$
\gamma(w)=\left[\begin{array}{c}
x(w) \\
x\left(w-\frac{h}{m}\right) \\
x\left(w-\frac{2 h}{m}\right) \\
\vdots \\
x\left(w-\frac{m-1}{m} h\right)
\end{array}\right] .
$$

The time derivative of $V(t)$ along the trajectory of the system in (13) is given by

$$
\begin{aligned}
& \dot{V}_{1}(t)=2 \dot{x}^{T}(t) P x(t) \\
& \dot{V}_{2}(t)=\frac{h}{m} \dot{x}^{T}(t) R(t) \dot{x}(t)-\int_{t-h / m}^{t} \dot{x}^{T}(w) R(w) \dot{x}(w) d w \\
& \dot{V}_{3}(t)=\gamma^{T}(t) Q(t) \gamma(t)-\gamma^{T}\left(t-\frac{h}{m}\right) Q\left(t-\frac{h}{m}\right) \gamma\left(t-\frac{h}{m}\right) .
\end{aligned}
$$

Define

$$
\xi(t)=\left[\begin{array}{c}
\gamma(t) \\
x(t-h) \\
\dot{x}(t)
\end{array}\right]
$$

and according to the Newton-Leibniz formula and the system in (12), with $S_{1}(t), S_{2}(t)$ defined in (14), we have

$$
\begin{aligned}
\Pi_{1}= & 2 \xi^{T}(t) S_{1}(t)\left[x(t)-x\left(t-\frac{h}{m}\right)-\int_{t-h / m}^{t} \dot{x}(w) d w\right]=0 \\
\Pi_{2}= & 2 \xi^{T}(t) S_{2}(t)\left[\left((A(t)+B(t) K(t)) x(t)+A_{d}(t) x(t-h)\right)\right. \\
& -\dot{x}(t)=0] .
\end{aligned}
$$

Therefore, the following holds:

$$
\begin{aligned}
\dot{V}(t) \leq & 2 \dot{x}^{T}(t) P x(t)+\frac{h}{m} \dot{x}^{T}(t) R(t) \dot{x}(t) \\
& -\int_{t-h / m}^{t} \dot{x}^{T}(w) R(w) \dot{x}(w) d w+\gamma^{T}(t) Q(t) \gamma(t) \\
& -\gamma^{T}\left(t-\frac{h}{m}\right) Q\left(t-\frac{h}{m}\right) \gamma\left(t-\frac{h}{m}\right) \\
& +2 \xi^{T}(t) S_{1}(t)\left[x(t)-x\left(t-\frac{h}{m}\right)-\int_{t-h / m}^{t} \dot{x}(w) d w\right] \\
& +2 \xi^{T}(t) S_{2}(t)[(A(t)+B(t) K(t)) x(t) \\
& \left.+A_{d}(t) x(t-h)-\dot{x}(t)\right] \\
& +\frac{h}{m} \xi^{T}(t) S_{1}(t) Z^{-1}(t) S_{1}^{T}(t) \xi(t) \\
& -\int_{t-h / m}^{t} \xi^{T}(t) S_{1}(t) Z^{-1}(t) S_{1}^{T}(t) \xi(t) d w
\end{aligned}
$$

where $Z(t)$ is to be determined.
If

$$
Z(t)<R(w)
$$

we have

$$
\begin{aligned}
\dot{V}(t) \leq & \Lambda(t)+\frac{h}{m} \xi^{T}(t) S_{1}(t) Z^{-1}(t) S_{1}^{T}(t) \xi(t) \\
& -\int_{t-h / m}^{t}\left(\dot{x}^{T}(w) R(w)+\xi^{T}(t) S_{1}(t)\right) \\
& \times R^{-1}(w)\left(R(w) \dot{x}(w)+S_{1}^{T}(t) \xi(t)\right) d w
\end{aligned}
$$

where

$$
\begin{aligned}
\Lambda(t)= & 2 \dot{x}^{T}(t) P x(t)+\frac{h}{m} \dot{x}^{T}(t) R(t) \dot{x}(t)+\gamma^{T}(t) Q(t) \gamma(t) \\
& -\gamma^{T}\left(t-\frac{h}{m}\right) Q\left(t-\frac{h}{m}\right) \gamma\left(t-\frac{h}{m}\right) \\
& +2 \xi^{T}(t) S_{1}(t)\left[x(t)-x\left(t-\frac{h}{m}\right)\right] \\
& +2 \xi^{T}(t) S_{2}(t)[(A(t)+B(t) K(t)) x(t) \\
& \left.+A_{d}(t) x(t-h)-\dot{x}(t)\right] \\
= & \xi^{T}(t) \Omega(t) \xi(t) .
\end{aligned}
$$

From (15), we know that

$$
\Omega(t)+\sigma W_{\sigma}^{T} W_{\sigma}+\frac{h}{m} S_{1}(t) Z^{-1}(t) S_{1}^{T}(t)<0 .
$$

Note that the last term in (20) is nonpositive, and

$$
\Omega(t)+\frac{h}{m} S_{1}(t) Z^{-1}(t) S_{1}^{T}(t)>-\sigma W_{\sigma}^{T} W_{\sigma} .
$$

Therefore

$$
\begin{aligned}
\dot{V}(t) & <\Lambda(t)+\frac{h}{m} \xi^{T}(t) S_{1}(t) Z^{-1}(t) S_{1}^{T}(t) \xi(t) \\
& <-\xi^{T}(t) \sigma W_{\sigma}^{T} W_{\sigma} \xi(t) .
\end{aligned}
$$

Thus, one can always find a sufficiently small $\sigma>0$, for $x(t) \neq$ 0 , satisfying

$$
\dot{V}(t)<-\sigma\|x(t)\|^{2}
$$

which indicates that the closed-loop fuzzy system is asymptotically stable, and the proof is completed.

It is noted that Theorem 1, expressed in the form of parameterdependent matrix inequalities, cannot be directly implemented for the stability analysis. Our next objective is to convert the inequalities in (15) and (16) to some finite LMIs, which can be readily solved using standard numerical software. We have the following theorem.

Theorem 2: Consider the nominal fuzzy time-delay system in (12) and suppose the controller gain matrices $K_{i}$ in (9) are known. Given an integer $m \geq 1$ and a scalar $h>0$, if there exist positive definite matrices $P, Q_{i}, Z_{i}$, and $R_{i}$, and matrices $S_{1 i}$ and $S_{2 i}$, for some positive scalar $\sigma$, satisfying

$$
\begin{aligned}
& \varphi_{i i l k}<0, \quad i, l, k=1, \ldots, r \\
& \varphi_{i j l k}+\varphi_{j i l k}<0, \quad 1 \leq i<j \leq r, \quad l, k=1, \ldots, r \\
& Z_{i}<R_{j}, \quad i, j=1, \ldots, r
\end{aligned}
$$


where

$$
\begin{aligned}
\varphi_{i j l k}= & {\left[\begin{array}{cc}
\Omega_{i j l k}+\sigma W_{\sigma}^{T} W_{\sigma} & S_{1 i} \\
* & -\frac{m}{h} Z_{i}
\end{array}\right] } \\
\Omega_{i j l k}= & W_{P}^{T} \bar{P} W_{P}+\frac{h}{m} W_{R}^{T} R_{i} W_{R}+W_{Q_{1}}^{T} Q_{i} W_{Q_{1}} \\
& -W_{Q_{2}}^{T} Q_{l} W_{Q_{2}}+\operatorname{sym}\left(S_{i} W_{S j k}\right) \\
S_{i}= & {\left[\begin{array}{ll}
S_{1 i} & S_{2 i}
\end{array}\right] } \\
W_{S j k}= & {\left[\begin{array}{cc}
I_{n}-I_{n} & 0_{n, m n} \\
A_{j}+B_{j} K_{k} 0_{n,(m-1) n} A_{d j}-I_{n}
\end{array}\right] }
\end{aligned}
$$

and $\bar{P}, W_{P}, W_{R}, W_{Q_{1}}, W_{Q_{2}}$, and $W_{\sigma}$ are defined in (17), then the fuzzy system in (12) is asymptotically stable.

Proof: Inequalities (15) and (16) can be rewritten as

$$
\begin{aligned}
& \sum_{i=1}^{r} \lambda_{i}(\theta(t)) \sum_{j=1}^{r} \lambda_{j}(\theta(t)) \sum_{l=1}^{r} \lambda_{l}\left(\theta\left(t-\frac{h}{m}\right)\right) \sum_{k=1}^{r} \lambda_{k}(\theta(t)) \varphi_{i j l k} \\
& =\sum_{i=1}^{r} \lambda_{i}^{2}(\theta(t)) \sum_{l=1}^{r} \lambda_{l}\left(\theta\left(t-\frac{h}{m}\right)\right) \sum_{k=1}^{r} \lambda_{k}(\theta(t)) \varphi_{i i l k} \\
& \quad+2 \sum_{i=1}^{r-1} \lambda_{i}(\theta(t)) \sum_{j>i}^{r} \lambda_{j}(\theta(t)) \sum_{l=1}^{r} \lambda_{l}\left(\theta\left(t-\frac{h}{m}\right)\right) \\
& \quad \times \sum_{k=1}^{r} \lambda_{k}(\theta(t))\left(\frac{\varphi_{i j l k}+\varphi_{j i l k}}{2}\right)<0
\end{aligned}
$$

and

$$
\sum_{i=1}^{r} \lambda_{i}(\theta(t)) \sum_{j=1}^{r} \lambda_{j}(\theta(w))\left(Z_{i}-R_{j}\right)<0 .
$$

Obviously, (21)-(23) guarantee the inequalities (15) and (16) for any $t$ and $w$.

The nominal fuzzy time-delay system in (6) with $u(t)=0$ can be described as

$$
\dot{x}(t)=\sum_{i=1}^{r} \lambda_{i}(\theta(t))\left[A_{i} x(t)+A_{d i} x(t-h)\right] .
$$

A new stability condition for this nominal unforced system is given in the following corollary, which can be proved by following similar arguments as those in the proof of Theorem 1.

Corollary 1: Given an integer $m \geq 1$ and a scalar $h>0$, the nominal fuzzy time-delay system in (12) when $u(t)=0$ is asymptotically stable if there exist positive definite matrices $P$, $Q_{i}, Z_{i}$, and $R_{i}$, and matrices $S_{1 i}$ and $S_{2 i}$, for some positive scalar $\sigma$, satisfying

$$
\begin{aligned}
& {\left[\begin{array}{cc}
\Omega_{i i l}+\sigma W_{\sigma}^{T} W_{\sigma} & S_{1 i} \\
* & -\frac{m}{h} Z_{i}
\end{array}\right]<0, \quad i, l=1, \ldots, r} \\
& {\left[\begin{array}{cc}
\Omega_{i j l}+\sigma W_{\sigma}^{T} W_{\sigma} & S_{1 i} \\
* & -\frac{m}{h} Z_{i}
\end{array}\right]+\left[\begin{array}{cc}
\Omega_{j i l}+\sigma W_{\sigma}^{T} W_{\sigma} & S_{1 j} \\
* & -\frac{m}{h} Z_{j}
\end{array}\right]<0} \\
& 1 \leq i<j \leq r, \quad l=1, \ldots, r \quad
\end{aligned}
$$

where

$$
\begin{aligned}
\Omega_{i j l}= & W_{P}^{T} \bar{P} W_{P}+\frac{h}{m} W_{R}^{T} R_{i} W_{R}+W_{Q_{1}}^{T} Q_{i} W_{Q_{1}} \\
& -W_{Q_{2}}^{T} Q_{l} W_{Q_{2}}+\operatorname{sym}\left(S_{i} \tilde{W}_{j}\right) \\
\tilde{W}_{j}= & {\left[\begin{array}{llll}
I_{n} & -I_{n} & 0_{n, m n} & \\
\hline A_{j} & 0_{n,(m-1) n} & A_{d j} & -I_{n}
\end{array}\right] }
\end{aligned}
$$

and $\bar{P}, S_{i}, W_{P}, W_{R}, W_{Q_{1}}, W_{Q_{2}}$, and $W_{\sigma}$ are the same as those in (24).

Remark 1: Theorem 1 and Corollary 1 present new stability conditions for fuzzy time-delay systems with or without control inputs, respectively. These conditions are derived based on the new Lyapunov-Krasovskii functional candidate defined in (18). It is worth noting that this Lyapunov-Krasovskii functional is essentially different from most of the existing ones used for fuzzy delay systems in that a new delay partitioning idea has been incorporated. Another feature worth mentioning is that in obtaining the new stability conditions, we have also incorporated the idea of basis dependence. These advanced ideas help reduce the conservatism, which will be well illustrated later in the Section VI.

Remark 2: Due to the incorporation of the idea of basis dependence, the stability conditions in Theorem 1 and Corollary 1 are large-computationally heavy, especially for systems with many fuzzy rules. If we do not utilize the basis-dependent idea, we can obtain Corollary 2, which is computationally more efficient.

Theorem 2 is developed based on a fuzzy basis-dependent Lyapunov functional. If we simplify it to the quadratic approach (i.e., fixed Lyapunov matrices are used for the whole fuzzy system), we obtain Corollary 2 given next. In this case, $Z$ is a redundant matrix variable (i.e., we do not need to introduce the matrix variable $Z$ ).

Corollary 2: Consider the nominal fuzzy time-delay system in (12) and suppose that the controller gain matrices $K_{i}$ in (9) are known. Given an integer $m \geq 1$ and a scalar $h>0$, if there exist positive definite matrices $P, Q$, and $R$, and matrices $S_{1}$ and $S_{2}$, for some positive scalar $\sigma$, satisfying

$$
\begin{aligned}
& {\left[\begin{array}{cc}
\Omega_{i i}+\sigma W_{\sigma}^{T} W_{\sigma} & S_{1} \\
* & -\frac{m}{h} R
\end{array}\right]<0, \quad i=1, \ldots, r} \\
& {\left[\begin{array}{cc}
\Omega_{i j}+\sigma W_{\sigma}^{T} W_{\sigma} & S_{1} \\
* & -\frac{m}{h} R
\end{array}\right]+\left[\begin{array}{cc}
\Omega_{j i}+\sigma W_{\sigma}^{T} W_{\sigma} & S_{1} \\
* & -\frac{m}{h} R
\end{array}\right]<0} \\
& 1 \leq i<j \leq r
\end{aligned}
$$

where

$$
\begin{aligned}
\Omega_{i j}= & W_{P}^{T} \bar{P} W_{P}+\frac{h}{m} W_{R}^{T} R W_{R}+W_{Q_{1}}^{T} Q W_{Q_{1}} \\
& -W_{Q_{2}}^{T} Q W_{Q_{2}}+\operatorname{sym}\left(\bar{S} W_{i j}\right) \\
\bar{S}= & {\left[\begin{array}{ll}
S_{1} & S_{2}
\end{array}\right] } \\
W_{i j}= & {\left[\begin{array}{llll}
\frac{I_{n}}{}-I_{n} & 0_{n, m n} & \\
\hline A_{i}+B_{i} K_{j} & 0_{n,(m-1) n} & A_{d i} & -I_{n}
\end{array}\right] }
\end{aligned}
$$


and $\bar{P}, W_{P}, W_{R}, W_{Q_{1}}, W_{Q_{2}}$, and $W_{\sigma}$ are defined in (17), then the fuzzy system in (12) is asymptotically stable.

Proof: The inequalities in (29) and (30) guarantee the following inequality holds:

$$
\left[\begin{array}{cc}
\Omega(t)+\sigma W_{\sigma}^{T} W_{\sigma} & S_{1} \\
* & -\frac{m}{h} R
\end{array}\right]<0
$$

where

$$
\begin{aligned}
\Omega(t)= & W_{P}^{T} \bar{P} W_{P}+\frac{h}{m} W_{R}^{T} R W_{R}+W_{Q_{1}}^{T} Q W_{Q_{1}} \\
& -W_{Q_{2}}^{T} Q W_{Q_{2}}+\operatorname{sym}(\bar{S} W(t)) \\
\bar{S}= & {\left[\begin{array}{lll}
S_{1} & S_{2}
\end{array}\right] } \\
W(t)= & {\left[\begin{array}{lll}
\frac{I_{n}}{A(t)+B(t) K(t)} & 0_{n,(m-1) n} & 0_{n, m n}(t)-I_{n}
\end{array}\right] . }
\end{aligned}
$$

The Lyapunov-Krasovskii functional candidate is introduced by simplifying the basis-dependent Lyapunov-Krasovskii functional candidate in Theorem 1 to quadratic one

$$
V(t)=V_{1}(t)+V_{2}(t)+V_{3}(t)
$$

and

$$
\begin{aligned}
& V_{1}(t)=x^{T}(t) P x(t) \\
& V_{2}(t)=\int_{-h / m}^{0} \int_{t+v}^{t} \dot{x}^{T}(w) R \dot{x}(w) d w d v \\
& V_{3}(t)=\int_{t-h / m}^{t} \gamma^{T}(w) Q \gamma(w) d w
\end{aligned}
$$

where $P>0, R>0$, and $Q>0$ are to be determined, and $\gamma(w)$ is the same as that in the proof of Theorem 1. By following similar lines as in Theorem 1, we obtain

$$
\begin{aligned}
\dot{V}(t) \leq & 2 \dot{x}^{T}(t) P x(t)+\frac{h}{m} \dot{x}^{T}(t) R \dot{x}(t)-\int_{t-h / m}^{t} \dot{x}^{T}(w) R \dot{x}(w) d w \\
& +\gamma^{T}(t) Q \gamma(t)-\gamma^{T}\left(t-\frac{h}{m}\right) Q \gamma\left(t-\frac{h}{m}\right) \\
& +2 \xi^{T}(t) S_{1}\left[x(t)-x\left(t-\frac{h}{m}\right)-\int_{t-h / m}^{t} \dot{x}(w) d w\right] \\
& +2 \xi^{T}(t) S_{2}[(A(t)+B(t) K(t)) x(t) \\
& \left.+A_{d}(t) x(t-h)-\dot{x}(t)\right]+\frac{h}{m} \xi^{T}(t) S_{1} R^{-1} S_{1}^{T} \xi(t) \\
& -\int_{t-h / m}^{t} \xi^{T}(t) S_{1} R^{-1} S_{1}^{T} \xi(t) d w
\end{aligned}
$$

i.e.,

$$
\begin{aligned}
\dot{V}(t) \leq & \Lambda(t)+\frac{h}{m} \xi^{T}(t) S_{1} R^{-1} S_{1}^{T} \xi(t)-\int_{t-h / m}^{t}\left(\dot{x}^{T}(w) R\right. \\
& \left.+\xi^{T}(t) S_{1}\right) R^{-1}\left(R \dot{x}(w)+S_{1}^{T} \xi(t)\right) d w
\end{aligned}
$$

where

$$
\begin{aligned}
\Lambda(t)= & 2 \dot{x}^{T}(t) P x(t)+\frac{h}{m} \dot{x}^{T}(t) R \dot{x}(t)+\gamma^{T}(t) Q \gamma(t) \\
& -\gamma^{T}\left(t-\frac{h}{m}\right) Q \gamma\left(t-\frac{h}{m}\right) \\
& +2 \xi^{T}(t) S_{1}\left[x(t)-x\left(t-\frac{h}{m}\right)\right] \\
& +2 \xi^{T}(t) S_{2}[(A(t)+B(t) K(t)) x(t) \\
& \left.+A_{d}(t) x(t-h)-\dot{x}(t)\right] \\
= & \xi^{T}(t) \Omega(t) \xi(t) .
\end{aligned}
$$

From (31), we know that

$$
\Omega(t)+\sigma W_{\sigma}^{T} W_{\sigma}+\frac{h}{m} S_{1} R^{-1} S_{1}^{T}<0 .
$$

By following similar arguments as those in the proof of Theorem 1, we can complete the proof.

\section{B. Uncertain Systems}

In this section, we consider the robust stability analysis of fuzzy time-delay systems with parameter uncertainties. The results for nominal systems in the previous section will be extended to systems with time-varying structured uncertainties described in (11). Before proceeding further, we first give the following lemma that is needed for our subsequent derivation.

Lemma 1 [37]: Given matrices $\Phi=\Phi^{T}, D, E$, and $R=$ $R^{T}>0$ of appropriate dimensions

$$
\Phi+D F E+E^{T} F^{T} D^{T}<0,
$$

for all $F$ satisfying $F^{T} F \leq R$, if and only if there exists a scalar $\varepsilon>0$ such that

$$
\Phi+\varepsilon D D^{T}+\varepsilon^{-1} E^{T} R E<0 .
$$

Theorem 3: Consider the fuzzy time-delay system in (11) and suppose that the controller gain matrices $K_{i}$ in (8) are known. Given an integer $m \geq 1$ and a scalar $h>0$, if there exist positive definite matrices $P, Q_{i}, Z_{i}$, and $R_{i}$, and matrices $S_{1 i}$ and $S_{2 i}$, scalars $\varepsilon_{i j l k}>0, \varepsilon_{i j l k}^{\prime}>0$, and $\varepsilon_{i j l k}^{\prime \prime}>0$, for some positive scalar $\sigma$, satisfying

$$
\begin{aligned}
& \psi_{i i l k}<0, \quad i, l, k=1, \ldots, r \\
& \psi_{i j l k}+\psi_{j i l k}<0, \quad 1 \leq i<j \leq r, \quad l, k=1, \ldots, r \\
& Z_{i}<R_{j}, \quad i, j=1, \ldots, r
\end{aligned}
$$

where

$$
\begin{aligned}
\psi_{i j l k}= & {\left[\begin{array}{ccccc}
\Theta_{i j l k} & S_{1 i} & S_{2 i} D_{a j} & S_{2 i} D_{d j} & S_{2 i} D_{b j} \\
* & -\frac{m}{h} Z_{i} & 0 & 0 & 0 \\
* & * & -\varepsilon_{i j l k} I_{n} & 0 & 0 \\
* & * & * & -\varepsilon_{i j l k}^{\prime} I_{n} & 0 \\
* & * & * & * & -\varepsilon_{i j l k}^{\prime \prime} I_{n}
\end{array}\right] } \\
\Theta_{i j l k}= & \Omega_{i j l k}+\sigma W_{\sigma}^{T} W_{\sigma}+\varepsilon_{i j l k} E_{A j}^{T} E_{A j} \\
& +\varepsilon_{i j l k}^{\prime} E_{D j}^{T} E_{D j}+\varepsilon_{i j l k}^{\prime \prime} E_{B j k}^{T} E_{B j k}
\end{aligned}
$$




$$
\begin{aligned}
E_{A i} & =\left[\begin{array}{ll}
E_{a i} & 0_{e_{1},(m+1) n}
\end{array}\right] \\
E_{B i j} & =\left[\begin{array}{ll}
E_{b i} K_{j} & 0_{e_{2},(m+1) n}
\end{array}\right] \\
E_{D i} & =\left[\begin{array}{lll}
0_{e_{3}, m n} & E_{d i} & 0_{e_{3}, n}
\end{array}\right]
\end{aligned}
$$

$\Omega_{i j l k}$ and $W_{\sigma}$ are defined in (24), and $e_{1}, e_{2}$, and $e_{3}$ are the numbers of rows in matrices $E_{a i}, E_{b i} K_{j}$, and $E_{d i}$, respectively, then the fuzzy system in (11) is asymptotically stable.

Proof: Replacing $A_{i}, B_{i}$ and $A_{d i}$ in (21) by $A_{i}+$ $D_{a i} F(t) E_{a i}, \quad B_{i}+D_{b i} F(t) E_{b i}, \quad$ and $\quad A_{d i}+D_{d i} F(t) E_{d i}$, respectively, we have

$$
\begin{array}{r}
\Omega_{i i l k}+\sigma W_{\sigma}^{T} W_{\sigma}+\frac{h}{m} S_{1 i} Z_{i}^{-1} S_{1 i}^{T}+\operatorname{sym}\left(\left(S_{2 i} D_{a i} F(t) E_{A i}\right.\right. \\
\left.+S_{2 i} D_{b i} F(t) E_{B i k}+S_{2 i} D_{d i} F(t) E_{D i}\right)<0 \\
i, l, k=1, \ldots, r .
\end{array}
$$

According to Lemma 1, the previous inequality holds if

$$
\begin{aligned}
\Omega_{i i l k} & +\sigma W_{\sigma}^{T} W_{\sigma}+\frac{h}{m} S_{1 i} Z_{i}^{-1} S_{1 i}^{T}+\varepsilon_{i i l k}^{-1} S_{2 i} D_{a i} D_{a i}^{T} S_{2 i}^{T} \\
& +\varepsilon_{i i l k}^{\prime-1} S_{2 i} D_{d i} D_{d i}^{T} S_{2 i}^{T}+\varepsilon_{i i l k}^{\prime \prime-1} S_{2 i} D_{b i} D_{b i}^{T} S_{2 i}^{T} \\
& +\varepsilon_{i i l k} E_{A i}^{T} E_{A i}+\varepsilon_{i i l k}^{\prime} E_{D i}^{T} E_{D i}+\varepsilon_{i i l k}^{\prime \prime} E_{B i k}^{T} E_{B i k}<0
\end{aligned}
$$

which, by Schur complement, is equivalent to the inequality in (33). By following similar lines, the inequality (34) can be obtained. The proof is completed.

The fuzzy time-delay systems with uncertainties in (11) when $u(t)=0$ can be described as

$$
\dot{x}(t)=\sum_{i=1}^{r} \lambda_{i}\left[\tilde{A}_{i} x(t)+\tilde{A}_{d i} x(t-h)\right] .
$$

Then, we have the following corollary, which can be proved by following similar arguments as those in the proof of Theorem 3.

Corollary 3: Given an integer $m \geq 1$ and a scalar $h>0$, the fuzzy time-delay system in (11) when $u(t)=0$ is asymptotically stable if there exist positive definite matrices $P, Q_{i}, Z_{i}$, and $R_{i}$, and matrices $S_{1 i}$ and $S_{2 i}$, scalars $\varepsilon_{i j l}>0$ and $\varepsilon_{i j l}^{\prime}>0$, for some positive scalar $\sigma$, satisfying

$$
\begin{aligned}
& \gamma_{i i l}<0, \quad i, l=1, \ldots, r \\
& \gamma_{i j l}+\gamma_{j i l}<0, \quad 1 \leq i<j \leq r, l=1, \ldots, r \\
& Z_{i}<R_{j}, \quad i, j=1, \ldots, r
\end{aligned}
$$

where

$$
\begin{aligned}
\gamma_{i j l} & =\left[\begin{array}{cccc}
\Theta_{i j l} & S_{1 i} & S_{2 i} D_{a j} & S_{2 i} D_{d j} \\
* & -\frac{m}{h} Z_{i} & 0 & 0 \\
* & * & -\varepsilon_{i j l} I_{n} & 0 \\
* & * & * & -\varepsilon_{i j l}^{\prime} I_{n}
\end{array}\right] \\
\Theta_{i j l} & =\Omega_{i j l}+\sigma W_{\sigma}^{T} W_{\sigma}+\varepsilon_{i j l} E_{A j}^{T} E_{A j}+\varepsilon_{i j l}^{\prime} E_{D j}^{T} E_{D j}
\end{aligned}
$$

and $\Omega_{i j l}$ and $W_{\sigma}$ are defined in (28).

\section{Controller Design}

In this section, fuzzy state-feedback controllers will be designed based on the results developed in the previous section. Delay-dependent stabilization methods will be developed such that the closed-loop fuzzy systems are asymptotically stable.

\section{A. Nominal Systems}

In this section, based on Theorem 1, a state-feedback controller is designed guaranteeing the asymptotic stability of the closed-loop nominal fuzzy system in (12).

Theorem 4: Consider the nominal fuzzy time-delay system in (6). Given an integer $m>1$, and scalars $h>0, \tau_{1}, \tau_{2}, \ldots$, $\tau_{(m+2)}$, there exists a fuzzy state-feedback controller in the form of (8) such that the closed-loop system in (12) is asymptotically stable if there exist positive definite matrices $\hat{P}, \hat{Q}_{i}, \hat{R}_{i}$, and $\hat{Z}_{i}$, and matrices $X, \hat{S}_{1 i}$, and $M_{i}$, for some positive scalar $\sigma$, satisfying

$$
\begin{gathered}
{\left[\begin{array}{ccc}
\Gamma_{i i l} & \hat{S}_{1 i} & W_{\sigma}^{T} X^{T} \\
* & -\frac{m}{h} \hat{Z}_{i} & 0 \\
* & * & -\sigma^{-1} I
\end{array}\right]<0, \quad i, l=1, \ldots r} \\
{\left[\begin{array}{ccc}
\Gamma_{i j l} & \hat{S}_{1 i} & W_{\sigma}^{T} X^{T} \\
* & -\frac{m}{h} \hat{Z}_{i} & 0 \\
* & * & -\sigma^{-1} I
\end{array}\right]+\left[\begin{array}{ccc}
\Gamma_{j i l} & \hat{S}_{1 j} & W_{\sigma}^{T} X^{T} \\
* & -\frac{m}{h} \hat{Z}_{j} & 0 \\
* & * & -\sigma^{-1} I
\end{array}\right]<0} \\
\hat{Z}_{i}<\hat{R}_{j}, \quad i, j=1, \ldots, r \\
\end{gathered}
$$

where

$$
\begin{aligned}
& \Gamma_{i j l}=W_{P}^{T} \check{P} W_{P}+\frac{h}{m} W_{R}^{T} \hat{R}_{i} W_{R}+W_{Q_{1}}^{T} \hat{Q}_{i} W_{Q_{1}} \\
& -W_{Q_{2}}^{T} \hat{Q}_{l} W_{Q_{2}}+\operatorname{sym}\left(G_{i} \hat{W}_{i j}\right) \\
& \check{P}=\left[\begin{array}{cc}
0 & \hat{P} \\
\hat{P} & 0
\end{array}\right], \quad G_{i}=\left[\begin{array}{ll}
S_{1 i} & U
\end{array}\right] \\
& U=\left[\begin{array}{llll}
\tau_{1} I_{n} & \tau_{2} I_{n} & \cdots & \tau_{(m+2)} I_{n}
\end{array}\right]^{T} \\
& \hat{W}_{i j}=\left[\begin{array}{cccc}
I_{n} & -I_{n} & 0_{n, m n} & \\
\hline A_{i} X+B_{i} M_{j} & 0_{n,(m-1) n} & A_{d i} X & -X
\end{array}\right]
\end{aligned}
$$

and $W_{P}, W_{R}, W_{Q_{1}}, W_{Q_{2}}$, and $W_{\sigma}$ are defined in (17). Furthermore, if the previous conditions are satisfied, the matrix gains of the controller are given by

$$
K_{i}=M_{i} X^{-1}, \quad i=1, \ldots, r .
$$

Proof: Suppose there exist positive definite matrices $\hat{P}, \hat{Q}_{i}$, $\hat{R}_{i}$, and $\hat{Z}_{i}$, and matrices $X, \hat{S}_{1 i}$, and $M_{i}$ satisfying the matrix inequalities in Theorem 4. Without the loss of generality, we assume that $X$ is invertible (invoke a small perturbation if necessary). Define $S=X^{-T}$ and the following matrices:

$$
\begin{aligned}
\bar{T} & =\operatorname{diag}\{S, S, \ldots S\} \in \mathbb{R}^{(m+4) n \times(m+4) n} \\
\bar{T}_{1} & =\operatorname{diag}\{S, S, \ldots, S\} \in \mathbb{R}^{(m+2) n \times(m+2) n} \\
\bar{T}_{2} & =\operatorname{diag}\{S, S, \ldots, S\} \in \mathbb{R}^{m n \times m n} .
\end{aligned}
$$


Pre- and postmultiplying (36) and (37) with $\bar{T}$ and $\bar{T}^{T}$, we obtain

$$
\begin{aligned}
& \bar{T}\left[\begin{array}{ccc}
\Gamma_{i i l} & \hat{S}_{1 i} & W_{\sigma}^{T} X^{T} \\
* & -\frac{m}{h} \hat{Z}_{i} & 0 \\
* & * & -\sigma^{-1} I
\end{array}\right] \bar{T}^{T}<0, \quad i, l=1, \ldots r \\
& \bar{T}\left[\begin{array}{ccc}
\Gamma_{i j l} & \hat{S}_{1 i} & W_{\sigma}^{T} X^{T} \\
* & -\frac{m}{h} \hat{Z}_{i} & 0 \\
* & * & -\sigma^{-1} I
\end{array}\right] \bar{T}^{T} \\
& +\bar{T}\left[\begin{array}{ccc}
\Gamma_{j i l} & \hat{S}_{1 j} & W_{\sigma}^{T} X^{T} \\
* & -\frac{m}{h} \hat{Z}_{j} & 0 \\
* & * & -\sigma^{-1} I
\end{array}\right] \bar{T}^{T}<0 \\
& 1 \leq i<j \leq r, \quad l=1, \ldots, r .
\end{aligned}
$$

Since

$$
\begin{aligned}
& \bar{T}\left[\begin{array}{ccc}
\Gamma_{i j l} & \hat{S}_{1 i} & W_{\sigma}^{T} X^{T} \\
* & -\frac{m}{h} \hat{Z}_{i} & 0 \\
* & * & -\sigma^{-1} I
\end{array}\right] \bar{T}^{T} \\
& =\left[\begin{array}{ccc}
\bar{T}_{1} & 0 & 0 \\
* & S & 0 \\
* & * & S
\end{array}\right]\left[\begin{array}{ccc}
\Gamma_{i j l} & \hat{S}_{1 i} & W_{\sigma}^{T} X^{T} \\
* & -\frac{m}{h} \hat{Z}_{i} & 0 \\
* & * & -\sigma^{-1} I
\end{array}\right] \\
& \times\left[\begin{array}{ccc}
\bar{T}_{1}^{T} & 0 & 0 \\
* & S^{T} & 0 \\
* & * & S^{T}
\end{array}\right] \\
& =\left[\begin{array}{ccc}
\bar{T}_{1} \Gamma_{i j l} \bar{T}_{1}^{T} & \bar{T}_{1} \hat{S}_{1 i} S^{T} & \bar{T}_{1} W_{\sigma}^{T} X^{T} S^{T} \\
* & -\frac{m}{h} S \hat{Z}_{i} S^{T} & 0 \\
* & * & -\sigma^{-1} S S^{T}
\end{array}\right]
\end{aligned}
$$

where

$$
\begin{aligned}
\bar{T}_{1} \Gamma_{i j l} \bar{T}_{1}^{T}= & W_{P}^{T}\left[\begin{array}{cc}
0 & S \hat{P} S^{T} \\
S \hat{P} S^{T} & 0
\end{array}\right] W_{P}+\frac{h}{m} W_{R}^{T} S \hat{R}_{i} S^{T} W_{R} \\
& +W_{Q_{1}}^{T} \bar{T}_{2} \hat{Q}_{i} \bar{T}_{2}^{T} W_{Q_{1}}-W_{Q_{2}}^{T} \bar{T}_{2} \hat{Q}_{l} \bar{T}_{2}^{T} W_{Q_{2}} \\
& +\operatorname{sym}\left(\bar{T}_{1} G_{i} \hat{W}_{i j} \bar{T}_{1}^{T}\right)
\end{aligned}
$$

and

$$
\bar{T}_{1} W_{\sigma}^{T} X^{T} S^{T}=W_{\sigma}^{T} S^{T}
$$

By defining

$$
\begin{aligned}
P & =S \hat{P} S^{T}, \quad R_{i}=S \hat{R}_{i} S^{T}, \quad Q_{i}=\bar{T}_{2} \hat{Q}_{i} \bar{T}_{2}^{T} \\
S_{1 i} & =\bar{T}_{1} \hat{S}_{1 i} S^{T}, \quad Z_{i}=S \hat{Z}_{i} S^{T} \\
S_{2 i} & =\left[\begin{array}{llll}
\tau_{1} S^{T} & \tau_{2} S^{T} & \cdots & \tau_{(m+2)} S^{T}
\end{array}\right]^{T}
\end{aligned}
$$

we have

$$
\begin{aligned}
\bar{T}_{1} \Gamma_{i j l} \bar{T}_{1}^{T}= & W_{P}^{T}\left[\begin{array}{cc}
0 & P \\
P & 0
\end{array}\right] W_{P}+\frac{h}{m} W_{R}^{T} R_{i} W_{R}+W_{Q_{1}}^{T} Q_{i} W_{Q_{1}} \\
& -W_{Q_{2}}^{T} Q_{l} W_{Q_{2}}+\operatorname{sym}\left(\bar{T}_{1} G_{i} \hat{W}_{i j} \bar{T}_{1}^{T}\right)
\end{aligned}
$$

where

$$
\begin{aligned}
& \bar{T}_{1} G_{i} \hat{W}_{i j} \bar{T}_{1}^{T} \\
= & \bar{T}_{1} \hat{S}_{1 i}\left[\begin{array}{llll}
I_{n} & -I_{n} & 0_{n, m n}
\end{array}\right] \bar{T}_{1}^{T} \\
& +\bar{T}_{1} U\left[\begin{array}{llll}
A_{i} X+B_{i} M_{j} & 0_{n,(m-1) n} & A_{d i} X & -X
\end{array}\right] \bar{T}_{1}^{T} \\
= & \bar{T}_{1} \hat{S}_{1 i} S^{T}\left[\begin{array}{llll}
I_{n} & -I_{n} & 0_{n, m n}
\end{array}\right] \\
& +\bar{T}_{1} U\left[\begin{array}{llll}
A_{i} X+B_{i} M_{j} & 0_{n,(m-1) n} & A_{d i} X & -X
\end{array}\right] \bar{T}_{1}^{T} \\
= & S_{1 i}\left[\begin{array}{lllll}
I_{n} & -I_{n} & 0_{n, m n}
\end{array}\right] \\
& +\left[\begin{array}{lllll}
\tau_{1} S^{T} & \tau_{2} S^{T} & \cdots & \tau_{(m+2)} S^{T}
\end{array}\right]^{T} \\
& \times\left[\begin{array}{lllll}
A_{i} X+B_{i} M_{j} & 0_{n,(m-1) n} & A_{d i} X & -X
\end{array}\right] \bar{T}_{1}^{T} \\
= & {\left[\begin{array}{lllll}
S_{1 i} & S_{2 i}
\end{array}\right]\left[\begin{array}{cccc}
I_{n}+B_{j} K_{k} & 0_{n,(m-1) n} & A_{d j} & -I_{n}
\end{array}\right] . }
\end{aligned}
$$

Thus, by Schur complement, we can obtain that

$$
\bar{T}\left[\begin{array}{ccc}
\Gamma_{i j l} & \hat{S}_{1 i} & W_{\sigma}^{T} X^{T} \\
* & -\frac{m}{h} \hat{Z}_{i} & 0 \\
* & * & -\sigma^{-1} I
\end{array}\right] \bar{T}^{T}<0
$$

is equivalent to

$$
\left[\begin{array}{cc}
\Omega_{i j l k}+\sigma W_{\sigma}^{T} W_{\sigma} & S_{1 i} \\
* & -\frac{m}{h} Z_{i}
\end{array}\right]<0 .
$$

Therefore, we conclude that the inequalities in (41) and (42) are equivalent to those in (21) and (22). Pre- and postmultiplying (38) with $S$ and $S^{T}$, we obtain (23). The proof is completed.

\section{B. Uncertain Systems}

In this section, we consider the problem of robust statefeedback controller design for the fuzzy delay systems with uncertainties in (1).

Theorem 5: Consider the fuzzy time-delay system in (1). Given an integer $m>1$, and scalars $h>0, \tau_{1}, \tau_{2}, \ldots, \tau_{(m+2)}$, there exists a fuzzy state-feedback controller in the form of (8) such that the closed-loop system in (11) is asymptotically stable if there exist positive definite matrices $\hat{P}, \hat{Q}_{i}, \hat{R}_{i}$, and $\hat{Z}_{i}$, matrices $X, \hat{S}_{1 i}$, and, $M_{i}$, and scalars $\epsilon_{i j l}>0, \epsilon_{i j l}^{\prime}>0$, and $\epsilon_{i j l}^{\prime \prime}>0$, for some positive scalar $\sigma$, satisfying

$$
\begin{aligned}
& \Xi_{i i l}<0, \quad i, l=1, \ldots r \\
& \Xi_{i j l}+\Xi_{j i l}<0, \quad 1 \leq i<j \leq r, \quad l=1, \ldots, r \\
& \hat{Z}_{i}<\hat{R}_{j}, \quad i, j=1, \ldots r
\end{aligned}
$$


where

$$
\begin{aligned}
\Xi_{i j l}= & {\left[\begin{array}{cccccc}
\tilde{\Gamma}_{i j l} & \hat{S}_{1 i} & W_{\sigma}^{T} X^{T} & \tilde{E}_{A i}^{T} & \tilde{E}_{B i j}^{T} & \tilde{E}_{D i}^{T} \\
* & -\frac{m}{h} \hat{Z}_{i} & 0 & 0 & 0 & 0 \\
* & * & -\sigma^{-1} I & 0 & 0 & 0 \\
* & * & * & -\varepsilon_{i j l} I_{n} & 0 & 0 \\
* & * & * & * & -\varepsilon_{i j l}^{\prime} I_{n} & 0 \\
* & * & * & * & * & -\varepsilon_{i j l}^{\prime \prime} I_{n}
\end{array}\right] } \\
\tilde{\Gamma}_{i j l}= & \Gamma_{i j l}+\varepsilon_{i j l}\left(U D_{a i}\right)\left(U D_{a i}\right)^{T} \\
& +\varepsilon_{i j l}^{\prime}\left(U D_{b i}\right)\left(U D_{b i}\right)^{T}+\varepsilon_{i j l}^{\prime \prime}\left(U D_{d i}\right)\left(U D_{d i}\right)^{T} \\
\tilde{E}_{A i}= & {\left[\begin{array}{lll}
E_{a i} X & 0_{f_{1},(m+1) n}
\end{array}\right] } \\
\tilde{E}_{B i j}= & {\left[\begin{array}{lll}
E_{b i} M M_{j} & 0_{f_{2},(m+1) n}
\end{array}\right] } \\
\tilde{E}_{D i}= & {\left[\begin{array}{lll}
0_{f_{3}, m n} & E_{d i} X & 0_{f_{3}, n}
\end{array}\right] }
\end{aligned}
$$

and $\Gamma_{i j l}, U$, and $W_{\sigma}$ are defined in (39), and $f_{1}, f_{2}$, and $f_{3}$ are the numbers of rows in matrices $E_{a i} X, E_{b i} M_{j}$, and $E_{d i} X$, respectively. Furthermore, if the previous conditions are satisfied, the matrix gains of the controller are given by

$$
K_{i}=M_{i} X^{-1}, \quad i=1, \ldots, r .
$$

Proof: Replacing $A_{i}, A_{d i}$, and $B_{i}$ in the inequalities (36) with

$$
A_{i}+D_{a i} F(t) E_{a i}(t), \quad A_{d i}+D_{d i} F(t) E_{d i}(t)
$$

and $B_{i}+D_{b i} F(t) E_{b i}(t)$, respectively, we get

$$
\begin{aligned}
\Gamma_{i i l} & +\frac{h}{m} \hat{S}_{1 i} \hat{Z}_{i}^{-1} \hat{S}_{1 i}^{T}+\sigma W_{\sigma}^{T} X^{T} X W_{\sigma}+\operatorname{sym}\left(U D_{a i} F(t) \tilde{E}_{A i}\right. \\
& \left.+U D_{b i} F(t) \tilde{E}_{B i j}+U D_{d i} F(t) \tilde{E}_{D i}\right)<0 .
\end{aligned}
$$

According to Lemma 1, the previous inequality holds if and only if

$$
\begin{aligned}
\Gamma_{i i l} & +\frac{h}{m} \hat{S}_{1 i} \hat{Z}_{i}^{-1} \hat{S}_{1 i}^{T}+\sigma W_{\sigma}^{T} X^{T} X W_{\sigma}+\epsilon_{i j l}\left(U D_{a i}\right)\left(U D_{a i}\right)^{T} \\
& +\epsilon_{i j l}^{\prime}\left(U D_{b i}\right)\left(U D_{b i}\right)^{T}+\epsilon_{i j l}^{\prime \prime}\left(U D_{d i}\right)\left(U D_{d i}\right)^{T} \\
& +\epsilon_{i j l}^{-1} \tilde{E}_{A i}^{T} \tilde{E}_{A i}+\epsilon_{i j l}^{\prime-1} \tilde{E}_{B i j}^{T} \tilde{E}_{B i j}+\epsilon_{i j l}^{\prime \prime T} \tilde{E}_{D i}^{T} \tilde{E}_{D i}<0 .
\end{aligned}
$$

By the Schur complement, the previous inequality is equivalent to (44). By following similar lines, the inequality (45) can be obtained, and the proof is completed.

Remark 3: Notice that there are some tuning parameters in the proposed controller design methods, and the values of these parameters are playing important roles with respect to the reduction of conservatism. To find a better combination of these parameters, one might resort to some optimization algorithms.

Remark 4: It is obvious that when the delay partitioning number $m$ becomes larger, the conservatism of the results is further reduced, while the computational cost increases. This is reasonable since $m$ is related to the number of decision variables. Thus, a larger $m$ implies that the solution can be searched in a larger set; on the other hand, it can be seen that the total number of decision variables is dependent on the delay partitioning number $m$, and it will increase if $m$ increases. For example, in Corollary 3 , the relation between the total number of decision variables $N$
TABLE I

Allowable MAXIMUM TIME DELAY $h$

\begin{tabular}{ccc}
\hline methods & $h_{\max }$ & $N$ \\
\hline$[7]$ & 0.7992 & \\
\hline$[28]$ & 3.1562 & \\
\hline$[34]$ & 3.3745 & \\
\hline$[40]$ & 3.8534 & \\
\hline Theorem 1, $m=2$ & 4.2832 & 61 \\
\hline Theorem 1, $m=3$ & 4.6221 & 91 \\
\hline Theorem 1, $m=4$ & 4.7108 & 129 \\
\hline Theorem 1, $m=10$ & 4.7369 & 525
\end{tabular}

and the delay partitioning number $m$ can be represented as

$$
N=N_{P}+N_{R_{i}}+N_{Z_{i}}+N_{Q_{i}}+N_{Q_{l}}+N_{S_{1 i}}+N_{S_{2 i}}
$$

where

$$
\begin{aligned}
N_{P} & =N_{R_{i}}=N_{Z_{i}}=\frac{n(n+1)}{2} \\
N_{Q_{i}} & =N_{Q_{l}}=\frac{m n(m n+1)}{2} \\
N_{S_{1 i}} & =N_{S_{2 i}}=(m+2) n n
\end{aligned}
$$

and $N_{P}, N_{R_{i}}, N_{Q_{i}}, N_{Q_{l}}, N_{S_{1 i}}$, and $N_{S_{2 i}}$ are the number of decision variables in $P, R_{i}, Q_{i}, Q_{l}, S_{1 i}$, and $S_{2 i}$.

\section{ILLUSTRATIVE EXAMPLE}

In this section, several examples are provided to demonstrate the effectiveness of the proposed approaches. By comparing with the results in the literature, the reduced conservatism of our methods becomes apparent. The first example is to show the advantage of the stability condition proposed in this paper. The second one is used to show the superiority of the proposed controller design methods. The third example is to show the applicability of the proposed controller design methods.

\section{A. Example 1}

Consider the following fuzzy system with a constant time delay, which has been used in many papers:

$$
\dot{x}(t)=\sum_{i=1}^{2} \lambda_{i}\left[A_{i} x(t)+A_{d i} x(t-h)\right]
$$

where

$$
\begin{aligned}
& A_{1}=\left[\begin{array}{cc}
-2.1 & 0.1 \\
-0.2 & -0.9
\end{array}\right], \quad A_{2}=\left[\begin{array}{cc}
-1.9 & 0 \\
-0.2 & -1.1
\end{array}\right] \\
& A_{d 1}=\left[\begin{array}{cc}
-1.1 & 0.1 \\
-0.8 & -0.9
\end{array}\right], \quad A_{d 2}=\left[\begin{array}{cc}
-0.9 & 0 \\
-1.1 & -1.2
\end{array}\right] \text {. }
\end{aligned}
$$

The purpose here is to find the allowable maximum time-delay value $h$ under which the fuzzy system is stable. Table I shows the maximum time-delay bounds obtained by the methods in [7], [30], [36], and [42] and that proposed by us, where " $N$ " stands for the total number of decision variables.

Table I clearly shows that our method yields much larger delay bounds than the existing result. It can also be seen from Table I that the conservatism is further reduced when $m$ increases. The advantage of our result is apparent. 
TABLE II

MAXIMUM TIME DELAY $h$ OF THE NOMINAL SYSTEM

\begin{tabular}{cc}
\hline methods & $h_{\max }$ \\
\hline$[28]$ & infeasible \\
\hline$[7]$ & 0.1524 \\
\hline$[34]$ & 0.2664 \\
\hline$[40]$ & 0.6611 \\
\hline Theorem 3 & 0.8420 \\
\hline
\end{tabular}

TABLE III

MAXIMUM Time Delay $h$ OF THE UNCERTAIN SySTEM

\begin{tabular}{cc}
\hline methods & $h_{\max }$ \\
\hline$h$ obtained by [7] & 0.1410 \\
\hline$h$ obtained by [40] & 0.1882 \\
\hline$h$ obtained by Theorem 4 & 0.3991 \\
\hline
\end{tabular}

\section{B. Example 2}

Consider the following fuzzy time-delay system with uncertainties:

$$
\begin{aligned}
\dot{x}(t)= & \sum_{i=1}^{r} \lambda_{i}\left[\left(A_{i}+\Delta A_{i}(t)\right) x(t)+A_{d i} x(t-h)\right. \\
& \left.+\left(B_{i}+\Delta B_{i}(t)\right) u(t)\right]
\end{aligned}
$$

where

$$
\begin{aligned}
A_{1} & =\left[\begin{array}{cc}
0 & 0.6 \\
0 & 1
\end{array}\right], \quad A_{2}=\left[\begin{array}{ll}
1 & 0 \\
1 & 0
\end{array}\right], \quad A_{d 1}=\left[\begin{array}{cc}
0.5 & 0.9 \\
0 & 2
\end{array}\right] \\
A_{d 2} & =\left[\begin{array}{cc}
0.9 & 0 \\
1 & 1.6
\end{array}\right], \quad B_{i}=\left[\begin{array}{l}
1 \\
1
\end{array}\right]
\end{aligned}
$$

and

$$
\begin{aligned}
\Delta A_{i}(t)=D F(t) E_{a i}, & \Delta A_{d i}(t)=D F(t) E_{d i} \\
\Delta B_{i}(t)=D F(t) E_{b i}, & F(t)=\sin (t)
\end{aligned}
$$

where

$$
\begin{aligned}
D & =\left[\begin{array}{l}
1 \\
0
\end{array}\right], \quad E_{a 1}=\left[\begin{array}{ll}
0 & 0
\end{array}\right] \\
E_{a 2} & =\left[\begin{array}{ll}
-0.05 & 0
\end{array}\right], \quad E_{b 1}=E_{b 2}=0.03 .
\end{aligned}
$$

First, we consider the nominal fuzzy system of (50). The model is the same as [36, Ex.2]. The approach in [30] fails to find stabilizing controllers. Table II shows the maximum time-delay values obtained by the controller design approaches in [7], [36], and [42] and the method presented in this paper. It is apparently shown that the time-delay bound obtained by our method is much larger than those obtained by others.

The fuzzy controller gains by our method are given by

$$
\begin{aligned}
& K_{1}=\left[\begin{array}{ll}
80.2956 & -217.9431
\end{array}\right] \\
& K_{2}=\left[\begin{array}{ll}
79.7132 & -218.4776
\end{array}\right] .
\end{aligned}
$$

Next, we consider robust control for the fuzzy system in (50). The results by applying the methods in [7] and [42] and Theorem 4 are listed in Table III. The reduction of conservatism given by our approach is clearly shown in Table III.

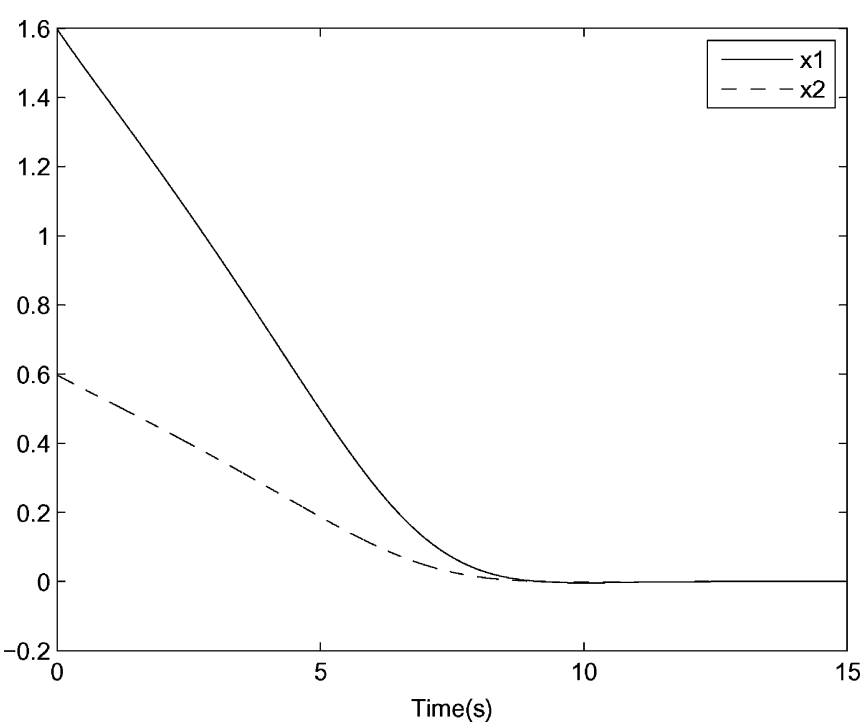

Fig. 1. State response of the nominal system.

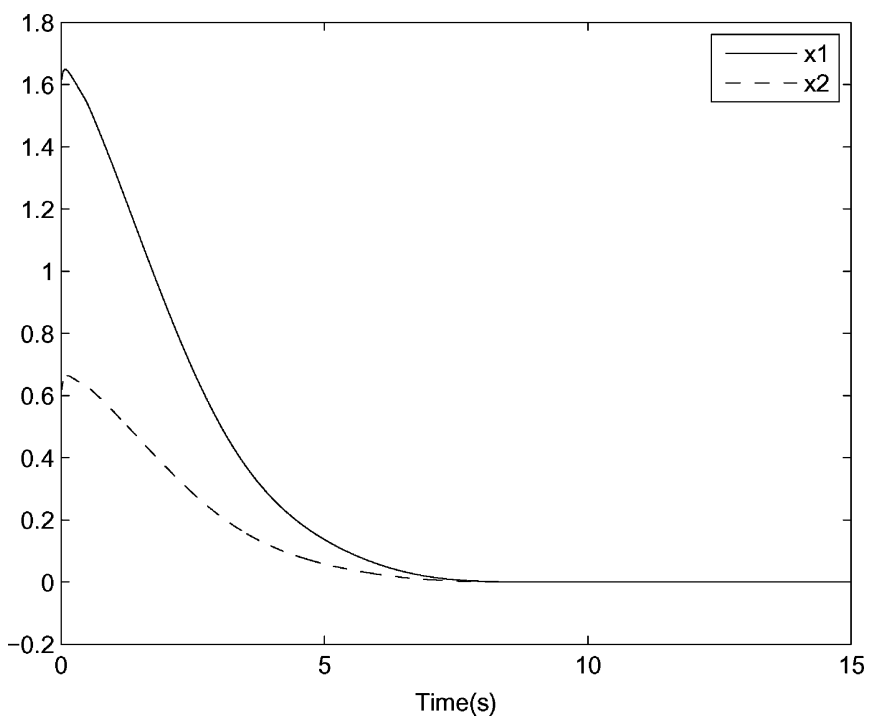

Fig. 2. State response of the uncertain system.

By Theorem 4 in this paper, for $h=0.3991$, the statefeedback gains are given by

$$
\begin{aligned}
& K_{1}=\left[\begin{array}{ll}
16.0804 & -42.6979
\end{array}\right] \\
& K_{2}=\left[\begin{array}{ll}
15.0867 & -42.9237
\end{array}\right] .
\end{aligned}
$$

In the simulation, we utilize the following fuzzy membership function [36]:

$$
\left\{\begin{array}{l}
\lambda_{1}\left(x_{1}(t)\right)=\frac{1}{\left(1+\exp \left(-2 x_{1}(t)\right)\right)} \\
\lambda_{2}\left(x_{1}(t)\right)=1-\lambda_{1}\left(x_{1}(t)\right) .
\end{array}\right.
$$

Fig. 1 shows that the states of the nominal closed-loop system converge to zero when the fuzzy controller gains are given in (51), and Fig. 2 shows the state response of the uncertain closed-loop system with the controller gains in (52), where 
the initial condition is assumed to be $x(t)=\left[\begin{array}{ll}1.6 & 0.6\end{array}\right]^{T}$, for $t \in[-0.3991,0]$.

\section{Example 3}

Consider the truck-trailer model with time delay formulated in [6]

$$
\begin{aligned}
& \dot{x}_{1}(t)=-c \frac{v \bar{t}}{L t_{0}} x_{1}(t)-(1-c) \frac{v \bar{t}}{L t_{0}} x_{1}(t-h)+\frac{v \bar{t}}{l t_{0}} u(t) \\
& \dot{x}_{2}(t)=c \frac{v \bar{t}}{L t_{0}} x_{1}(t)+(1-c) \frac{v \bar{t}}{L t_{0}} x_{1}(t-h) \\
& \dot{x}_{3}(t)=\frac{v \bar{t}}{t_{0}} \sin \left(x_{2}(t)+c \frac{v \bar{t}}{2 L} x_{1}(t)+(1-c) \frac{v \bar{t}}{2 L} x_{1}(t-h)\right)
\end{aligned}
$$

where $x_{1}(t)$ is the angular difference between the truck and trailer, $x_{2}(t)$ is the angle of the trailer, and $x_{3}(t)$ is the vertical position of rear end of the trailer. The model parameters are given as $l=2.8, L=5.5, v=-1.0, \bar{t}=2.0, t_{0}=0.5$, and $c=0.7$.

Let $\theta(t)=x_{2}(t)+c(v \bar{t} / 2 L) x_{1}(t)+(1-c)(v \bar{t} / 2 L) x_{1}(t-$ $h)$, and the membership functions are defined in [36]. The T-S fuzzy model that represents the nonlinear system is as follows:

Model rule 1: IF $\theta(t)$ is about $0 \mathrm{rad}$

$$
\text { THEN } \dot{x}(t)=A_{1} x(t)+A_{d 1} x(t-h)+B_{1} u(t)
$$

Model rule 2: IF $\theta(t)$ is about $\pi \mathrm{rad}$ or $-\pi \mathrm{rad}$

$$
\text { THEN } \dot{x}(t)=A_{2} x(t)+A_{d 2} x(t-h)+B_{2} u(t)
$$

where $x(t)=\left[\begin{array}{lll}x_{1}(t) & x_{2}(t) & x_{3}(t)\end{array}\right]^{T}$, and

$$
\begin{aligned}
A_{1} & =\left[\begin{array}{ccc}
-c \frac{v \bar{t}}{L t_{0}} & 0 & 0 \\
c \frac{v \bar{t}}{L t_{0}} & 0 & 0 \\
c \frac{v^{2} \bar{t}^{2}}{2 L t_{0}} & \frac{v \bar{t}}{t_{0}} & 0
\end{array}\right], \quad A_{d 1}=\left[\begin{array}{ccc}
-(1-c) \frac{v \bar{t}}{L t_{0}} & 0 & 0 \\
(1-c) \frac{v \bar{t}}{L t_{0}} & 0 & 0 \\
(1-c) \frac{v^{2} \bar{t}^{2}}{2 L t_{0}} & 0 & 0
\end{array}\right] \\
A_{2} & =\left[\begin{array}{ccc}
-c \frac{v \bar{t}}{L t_{0}} & 0 & 0 \\
c \frac{v \bar{t}}{L t_{0}} & 0 & 0 \\
c \frac{g v^{2} \bar{t}^{2}}{2 L t_{0}} & \frac{g v \bar{t}}{t_{0}} & 0
\end{array}\right], \quad A_{d 2}=\left[\begin{array}{ccc}
-(1-c) \frac{v \bar{t}}{L t_{0}} & 0 & 0 \\
(1-c) \frac{v \bar{t}}{L t_{0}} & 0 & 0 \\
(1-c) \frac{g v^{2} \bar{t}^{2}}{2 L t_{0}} & 0 & 0
\end{array}\right] \\
B_{1} & =\left[\begin{array}{ccc}
\frac{v \bar{t}}{l t_{0}} & 0 & 0
\end{array}\right]^{T}, \quad B_{2}=\left[\begin{array}{lll}
\frac{v \bar{t}}{l t_{0}} & 0 & 0
\end{array}\right]^{T} .
\end{aligned}
$$

We assume that the uncertainties in the system are modeled as that in [8], where $F(t)=\sin (2 t)$, and

$$
\begin{aligned}
D_{a} & =D_{d}=\left[\begin{array}{lll}
0.255 & 0.255 & 0.255
\end{array}\right]^{T} \\
E_{a 1} & =E_{a 2}=E_{d 1}=E_{d 2}=\left[\begin{array}{lll}
0.1 & 0 & 0
\end{array}\right] \\
D_{b} & =\left[\begin{array}{lll}
0.1790 & 0 & 0
\end{array}\right]^{T}, \quad E_{b 1}=0.05, \quad E_{b 2}=0.15 .
\end{aligned}
$$
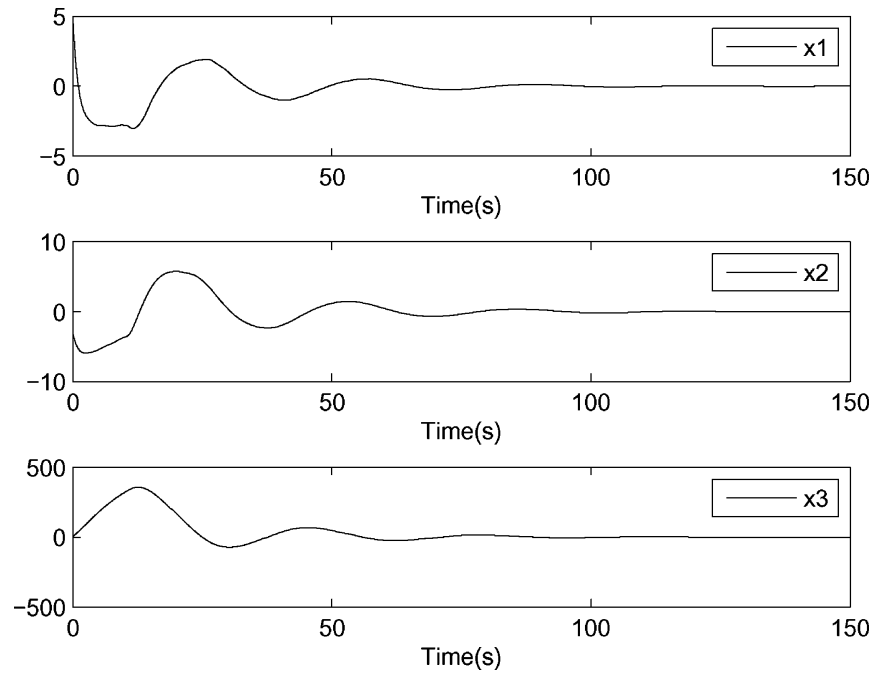

Fig. 3. State response for Example 3.

By using Theorem 4, we found that the fuzzy system is robustly stabilizable with the maximum time-delay value $h=10.4653$, and the fuzzy state-feedback controller gains are given by

$$
\begin{aligned}
& K_{1}=\left[\begin{array}{lll}
1.2896 & -0.4511 & 0.0052
\end{array}\right] \\
& K_{2}=\left[\begin{array}{lll}
1.2912 & -0.4602 & 0.0052
\end{array}\right] .
\end{aligned}
$$

The obtained fuzzy state-feedback controller makes the closedloop states converge to zero, as is shown in Fig. 3, where the initial condition is assumed to be $x(t)=\left[\begin{array}{lll}5 & -3 & 4\end{array}\right]^{T}$, for $t \in[-10.4653,0]$.

\section{CONCLUDING REMARKS}

The problems of delay-dependent stability analysis and stabilization of Takagi-Sugeno fuzzy time-delay systems have been investigated. Based on the delay fractioning technique, a new approach has been proposed, with elegant delay-dependent stability condition derived for the nominal fuzzy delay systems. This result has been further utilized to solve the stabilization problem. The results for the nominal system have also been extended to deal with the robust stability analysis and stabilization problems for fuzzy delay systems with time-varying parameter uncertainties. Several examples have been provided to show the effectiveness and advantage of the obtained results. The delay partititioning idea has been well demonstrated to be efficient for reducing conservatism and could be further extended to solve other related problems.

\section{REFERENCES}

[1] W. Assawinchaichote and S. K. Nguang, " $H_{\infty}$ filtering for fuzzy singularly perturbed systems with pole placement constraints: An LMI approach," IEEE Trans. Signal Process., vol. 52, no. 6, pp. 1659-1667, Jun. 2004.

[2] M. Basin and M. Skliar, "Integral approach to optimal filtering and control of continuous processes with time-varying delays," in Proc. 40th Conf. Decis. Control, Orlando, FL, 2001, pp. 2911-2916.

[3] E. K. Boukas and N. F. Al-Muthairi, "Delay-dependent stabilization of singular linear systems with delays," Int. J. Innovative Comput., Inf. Control, vol. 2, no. 2, pp. 283-291, 2006. 
[4] S. G. Cao, N. W. Rees, and G. Feng, "Analysis and design of a class of continuous time fuzzy control systems," Int. J. Control, vol. 64, pp. 10691087,1996

[5] Y. Y. Cao and P. M. Frank, "Robust $H_{\infty}$ disturbance attenuation for a class of uncertain discrete-time fuzzy systems," IEEE Trans. Fuzzy Syst., vol. 8, no. 4, pp. 406-415, Aug. 2000.

[6] Y. Y. Cao and P. M. Frank, "Stability analysis and synthesis of nonlinear time-delay systems via linear Takagi-Sugeno fuzzy models," Fuzzy Sets Syst., vol. 124, pp. 213-229, 2001.

[7] B. Chen and X. P. Liu, "Delay-dependent robust $H_{\infty}$ control for T-S fuzzy systems with time delay," IEEE Trans. Fuzzy Syst., vol. 13, no. 4, pp. 544-556, Aug. 2005

[8] B. Chen, X. P. Liu, and S. C. Tong, "New delay-dependent stabilization conditions of T-S fuzzy systems with constant delay," Fuzzy Sets Syst., vol. 158, no. 20, pp. 2209-2224, 2007.

[9] C. E. de Souza and X. Li, "Delay-dependent robust $H_{\infty}$ control of uncertain linear state-delayed systems," Automatica, vol. 35, pp. 1313-1321, 1999.

[10] G. Feng, "Robust $H_{\infty}$ filtering of fuzzy dynamic systems," IEEE Trans. Aerosp. Electron. Syst., vol. 41, no. 2, pp. 658-671, Apr. 2005.

[11] G. Feng, "A survey on analysis and design of model-based fuzzy control systems," IEEE Trans. Fuzzy Syst., vol. 14, no. 5, pp. 676-697, Oct. 2006

[12] E. Fridman and U. Shaked, "Delay-dependent stability and $H_{\infty}$ control: Constant and time-varying delays," Int. J. Control, vol. 76, no. 1, pp. 4860, 2003 .

[13] H. Gao and C. Wang, "Delay-dependent robust $H_{\infty}$ and $L_{2}-L_{\infty}$ filtering for a class of uncertain nonlinear time-delay systems," IEEE Trans. Autom. Control, vol. 48, no. 9, pp. 1661-1666, Sep. 2003.

[14] H. Gao and C. Wang, "A delay-dependent approach to robust $H_{\infty}$ filtering for uncertain discrete-time state-delayed systems," IEEE Trans. Signal Process., vol. 52, no. 6, pp. 1631-1640, Jun. 2004.

[15] H. Gao, Z. Wang, and C. Wang, "Improved $H_{\infty}$ control of discrete-time fuzzy systems: A cone complementarity linearization approach," Inf. Sci., vol. 175 , no. 1/2, pp. 57-77, 2005.

[16] F. Gouaisbaut and D. Peaucelle, "Delay-dependent stability analysis of linear time delay systems," in Proc. IFAC TDC, 2006, pp. 5-7.

[17] Y. He, Q. G. Wang, and C. Lin, "An improved $H_{\infty}$ filter design for systems with time-varying interval delay," IEEE Trans. Circuits Syst. II, Exp. Briefs, vol. 53, no. 11, pp. 1235-1239, Nov. 2006.

[18] Y. He, M. Wu, J. H. She, and G. P. Liu, "Parameter-dependent Lyapunov functional for stability of time-delay systems with polytopic-type uncertainties," IEEE Trans. Autom. Control, vol. 49, no. 5, pp. 828-832, May 2004.

[19] E. Kim and H. Lee, "New approaches to relaxed quadratic stability condition of fuzzy control systems," IEEE Trans. Fuzzy Syst., vol. 8, no. 5, pp. 523-534, Oct. 2000.

[20] J. Lam, H. Gao, and C. Wang, "Stability analysis for continuous systems with two additive time-varying delay components," Syst. Control Lett., vol. 56, no. 1, pp. 16-24, 2007.

[21] J. Lam and S. S. Zhou, "Dynamic output feedback $H_{\infty}$ control of discretetime fuzzy systems: A fuzzy-basis-dependent Lyapunov function approach," Int. J. Syst. Sci., vol. 38, no. 1, pp. 25-37, 2007.

[22] C. Lin, Q. G. Wang, and T. H. Lee, "Improvement on observer-based $H_{\infty}$ control for T-S fuzzy systems," Automatica, vol. 41, no. 9, pp. 1651-1656, 2005.

[23] C. Lin, Q. G. Wang, and T. H. Lee, "Stabilization of uncertain fuzzy time-delay systems via variable structure control approach," IEEE Trans. Fuzzy Syst., vol. 13, no. 6, pp. 787-798, Dec. 2005.

[24] C. Lin, Q. G. Wang, and T. H. Lee, "Stability and stabilization of a class of fuzzy time-delay descriptor systems," IEEE Trans. Fuzzy Syst., vol. 14, no. 4, pp. 542-551, Aug. 2006.

[25] S. K. Nguang, P. Shi, and S. Ding, "Delay-dependent fault estimation for uncertain time-delay nonlinear systems: An LMI approach," Int. J. Robust Nonlinear Control, vol. 16, no. 18, pp. 913-933, 2006.

[26] P. Shi, "Filtering on sampled-data systems with parametric uncertainty," IEEE Trans. Autom. Control, vol. 43, no. 7, pp. 1022-1027, Jul. 1998.

[27] P. Shi, E. K. Boukas, and R. K. Agarwal, "Control of Markovian jump discrete-time systems with norm bounded uncertainty and unknown delay," IEEE Trans. Autom. Control, vol. 44, no. 11, pp. 2139-2144, Nov. 1999.

[28] P. Shi and S. K. Nguang, " $H_{\infty}$ output feedback control of fuzzy system models under sampled measurements," Comput. Math. Appl., vol. 46, no. 5-6, pp. 705-717, 2003.
[29] K. Tanaka, T. Ikeda, and H. O. Wang, "Fuzzy regulators and fuzzy observers: Relaxed stability conditions and LMI-based designs," IEEE Trans. Fuzzy Syst., vol. 6, no. 5, pp. 250-265, May 1998.

[30] E. Tian and C. Peng, "Delay-dependent stabilization analysis and synthesis of uncertain T-S fuzzy systems with time-varying delay," Fuzzy Sets Syst., vol. 157, pp. 544-559, 2006.

[31] H. O. Wang, K. Tanaka, and M. F. Griffin, "An approach to fuzzy control of nonlinear systems: Stability and design issues," IEEE Trans. Fuzzy Syst., vol. 4, no. 2, pp. 14-23, Feb. 1996.

[32] Z. Wang, D. W. C. Ho, and X. Liu, "A note on the robust stability of uncertain stochastic fuzzy systems with time-delays," IEEE Trans. Syst., Man, Cybern. A, Syst., Humans, vol. 34, no. 4, pp. 570-576, Jul. 2004.

[33] Z. Wang, J. Lam, and X. H. Liu, "Nonlinear filtering for state delayed systems with Markovian switching," IEEE Trans. Signal Process., vol. 51, no. 9, pp. 2321-2328, Sep. 2003.

[34] H. Wu, "Reliable LQ fuzzy control for continuous-time nonlinear systems with actuator faults," IEEE Trans. Syst., Man, Cybern. B, Cybern, vol. 34 no. 2, pp. 1743-1752, Apr. 2004.

[35] H. Wu, "Reliable LQ fuzzy control for nonlinear discrete-time systems via LMIs," IEEE Trans. Syst., Man, Cybern. B, Cybern, vol. 34, no. 2 , pp. 1270-1275, Apr. 2004

[36] H. Wu and H. X. Li, "New approach to delay-dependent stability analysis and stabilization for continuous-time fuzzy control systems with timevarying delay," IEEE Trans. Fuzzy Syst., vol. 15, no. 3, pp. 482-493, Jun 2007.

[37] L. Xie, "Output feedback $H_{\infty}$ control of systems with parameter uncertainty," Int. J. Control, vol. 63, pp. 741-750, 1996.

[38] L. Xie, E. Fridman, and U. Shaked, "Robust $H_{\infty}$ control of distributed delay systems with application to combustion control," IEEE Trans. Autom. Control, vol. 46, no. 12, pp. 1930-1935, Dec. 2001.

[39] B. Xu and Y. Liu, "Delay-dependent/delay-independent stability of linear systems with multiple time-varying delays," IEEE Trans. Autom. Control, vol. 48, no. 4, pp. 697-701, Apr. 2003.

[40] S. Xu and J. Lam, "Robust $H_{\infty}$ control for uncertain discrete time-delay fuzzy systems via output feedback controllers," IEEE Trans. Fuzzy Syst. vol. 13, no. 1, pp. 82-93, Feb. 2005

[41] S. Xu, J. Lam, S. Huang, and C. Yang, " $H_{\infty}$ model reduction for linear time-delay systems: Continuous-time case," Int. J. Control, vol. 74, no. 11, pp. 1062-1074, 2001.

[42] J. Yoneyama, "New delay-dependent approach to robust stability and stabilization for Takagi-Sugeno fuzzy time-delay systems," Fuzzy Sets Syst., vol. 158, no. 2, pp. 2225-2237, 2007.

[43] D. Yue, J. Fang, and S. Won, "Delay-dependent robust stability of stochastic uncertain systems with time delay and Markovian jump parameters," Circuits, Syst. Signal Process., vol. 22, pp. 351-365, 2003.

[44] D. Yue and Q.-L. Han, "Robust $H_{\infty}$ filter design of uncertain descriptor systems with discrete and distributed delays," IEEE Trans. Signal Process., vol. 52, no. 11, pp. 3200-3212, Nov. 2004.

[45] S. Zhou, G. Feng, J. Lam, and S. Xu, "Robust $H_{\infty}$ control for discretetime fuzzy systems via basis-dependent Lyapunov functions," Inf. Sci., vol. 174, no. 3/4, pp. 197-217, 2005.

[46] S. Zhou, J. Lam, and A. K. Xue, " $H_{\infty}$ filtering of discrete-time fuzzy systems via basis-dependent Lyapunov function approach," Fuzzy Sets Syst., vol. 158, pp. 180-193, 2007.

[47] S. Zhou and T. Li, "Robust stabilization for delayed discrete-time fuzzy systems via basis-dependent Lyapunov-Krasovskii function," Fuzzy Sets Syst., vol. 151, no. 1, pp. 139-153, 2005.

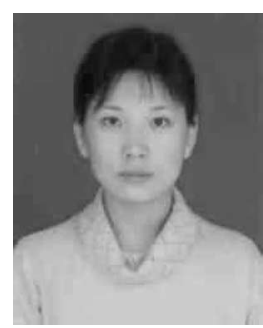

Yan Zhao received the B.S. degree in chemical engineering and equipment control and the M.S. degree in mechanical engineering from Inner Mongolia University of Technology, Hohhot, China, in 2002 and 2005 , respectively. She is currently working toward the $\mathrm{Ph} . \mathrm{D}$. degree in control science and engineering with Harbin Institute of Technology, Harbin, China.

Her current research interests include fuzzy control systems, robust control, and networked control systems. 


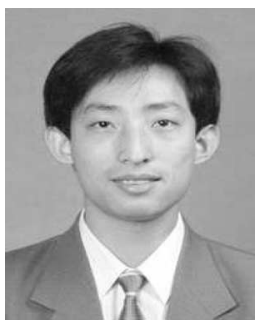

Huijun Gao (M'05) was born in Heilongjiang Province, China, in 1976. He received the M.S. degree in electrical engineering from Shenyang University of Technology, Shengyang, China, in 2001 and the $\mathrm{Ph} . \mathrm{D}$ degree in control science and engineering from Harbin Institute of Technology, Harbin, China, in 2005 .

From November 2003 to August 2004, he was a Research Associate with the Department of Mechanical Engineering, University of Hong Kong, Pok Fu Lam, Hong Kong. From October 2005 to September 2007, he was a Postdoctoral Researcher with the Department of Electrical and Computer Engineering, University of Alberta, Edmonton, AB, Canada, supported by an Alberta Ingenuity Fellowship and an Honorary Izaak Walton Killam Memorial Postdoctoral Fellowship. He is currently a Professor with Harbin Institute of Technology. His current research interests include networkbased control, robust control/filter theory, model reduction, time-delay systems, and multidimensional systems and their engineering applications. He is an Associate Editor of the Journal of Intelligent and Robotics Systems and the International Journal of Innovative Computing, Information and Control and serves on the Editorial Board of the International Journal of Systems Science and the Journal of the Franklin Institute and Nonlinear Dynamics and Systems Theory.

Prof. Gao is an Associate Editor of the IEEE TRANSACTIONS ON SYSTEMS, MAN, AND CYBERNETICS, PART B: CYBERNETICS and IEEE TRANSACTIONS ON INDUSTRIAL ELECTRONICS. He was the recipient of the University of Alberta Dorothy J. Killam Memorial Postdoctoral Fellow Prize in 2005 and was a corecipient of the Outstanding Science and Technology Development Awards from the Ministry of Machine-Building Industry of China and from the Liaoning Provincial Government of China, both in 2002.

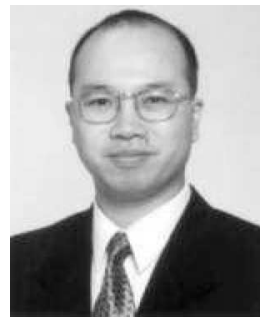

James Lam (S'86-M'87-SM'99) received the B.Sc. degree (with first class) in mechanical engineering from the University of Manchester, Manchester, U.K., in 1983, and the M.Phil. and Ph.D. degrees in control engineering from the University of Cambridge, Cambridge, U.K., in 1985 and 1988, respectively. He received the Ashbury Scholarship, the A.H. Gibson Prize, and the H. Wright Baker Prize for his academic performance.

He is currently a Professor with the Department of Mechanical Engineering, University of Hong Kong, Hong Kong. He is an Associate Editor of the Asian Journal of Control, the International Journal of Systems Science, the Journal of Sound and Vibration, the International Journal of Applied Mathematics and Computer Science, the IEEE TRANSACTIONS ON SigNAL PROCESSING, the Journal of the Franklin Institute, Dynamics of Continuous, Discrete, and Impulsive Systems (Series B: Applications and Algorithms), and Automatica. He is also a member of the Editorial Board of the IET Control Theory and Applications, Open Electrical and Electronic Engineering Journal, Research Letters in Signal Processing, the International Journal of Systems, Control, and Communications, and the Journal of Electrical and Computer Engineering. His current research interests include reduced-order modeling, delay systems, descriptor systems, stochastic systems, multidimensional systems, robust control, and ltering. He was an Editor-in-Chief of Proceedings of the IEE Control Theory and Applications.

Prof. Lam is a Chartered Mathematician and a Chartered Scientist. He is a Fellow of the Institute of Mathematics and Its Applications, and the IET. He is a Scholar and a Fellow of the Croucher Foundation. He is a Conference Editorial Board member of the IEEE Control Systems Society.

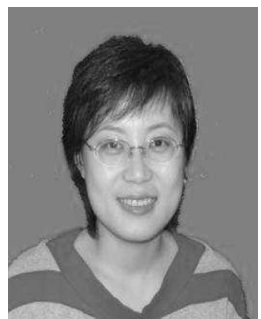

Baozhu Du received the B.S. degree in information and computing science, and the M.S. degree in operational research and cybernetics from Northeastern University, Shenyang, Liaoning Province, China, in 2003 and 2006, respectively. She is currently working toward the Ph.D. degree in mechanical engineering with the University of Hong Kong, Pok Fu Lam, Hong Kong.

Her current research interests include stability analysis and robust control/filter theory of time-delay systems. 